\title{
VỀ VĂN HÓA GIAO TIẾP CỦA NGƯỜI VIỆT \\ QUA VIỆC SỬ DỤNG HƯ TỬ MANG NGHĨA HÀM ẨN
}

Vietnamese communicative culture through the use of functional words with implicature

Ngày nhận bài: 01/9/2016; ngày phản biện: 15/10/2016; ngày duyệt đăng: 21/11/2016

\section{Bùi Minh Toán*}

\section{TÓM TẮT}

Hư từ trong ngôn ngữ nói chung và trong tiếng Việt nói riêng, tuy không thực hiện được chức năng định danh, không đảm nhiệm các chức năng ngữ pháp chính trong cụm từ và trong câu, nhưng đối với việc thể hiện nghĩa tình thái trong câu và đối với việc tạo lập câu mang nghĩa hàm ẩn thì nó lại là một phương tiện có giá trị không nhỏ. Báo cáo phân tích vai trò của một số lớp hư từ thuộc phụ từ, tình thái từ, quan hệ từ trong tiếng Việt đối với việc thể hiện nghĩa hàm ẩn trong câu. Hơn nữa, phần nghĩa hàm ẩn đó có thể thuộc lĩnh vực nghĩa miêu tả hay nghĩa tình thái. Qua đó, bài báo góp phần khẳng định vai trò của hư từ tiếng Việt trong văn hóa giao tiếp bằng ngôn ngữ của người Việt.

Từ khóa: hư tì̀; nghĩa hàm ẩn; nghĩa tình thái; nghĩa miêu tả; văn hóa giao tiếp

\section{ABSTRACT}

Functional words in languages in general, and in Vietnamese in particular, are means of no trivial values to the representation of modality in sentences as well as the formulation of sentences with implicature, even though they do not serve any nomenclature nor fundamental syntactic functions in phrases or sentences. This paper analyses the roles of several classes of functional words, including particles, modals and relationals in Vietnamese in expressing implicature in sentences. Such implicature, moreover, can be part of descriptive/representational meaning, or modal meaning. The analysis presented in the paper helps assert the roles of Vietnamese functional words in Vietnamese communicative culture via the means of language.

Keywords: functional words; implicature; modal meaning; descriptive/representational meaning; communicative culture

Báo cáo xuất phát từ quan niệm phổ biến về nghĩa của câu. Theo đó, nghĩa của câu được phân biệt theo tính chất thành hai thành phần: nghĩa miêu tả và nghĩa tình thái, còn theo cách thức biểu hiện thì phân biệt thành nghĩa tường minh và nghĩa hàm ẩn. Nghĩa miêu tả là nội dung đề cập đến một sự tình nào đó mà con người nhận thức được. Nó luôn luôn gắn liền với nghĩa tình thái, trong đó phần quan trọng là thái độ, sự nhìn nhận, đánh giá của người nói đối với sự tình. Cho nên nghĩa tình thái vừa thể hiện điểm nhìn của cá nhân người nói, vừa mang theo dấu ấn văn hóa của cộng đồng xã hội mà người nói là thành viên. Mặt khác, trong giao tiếp ngôn ngữ, con người có thể lựa chọn cách thức biểu hiện tường minh hay hàm ẩn. Với phát ngôn mang nghĩa hàm ẩn (tiền giả định và hàm ý), lời nói vừa súc tích, ngắn gọn, vừa tế nhị, lịch sự, lại có thể đạt hiệu quả giao tiếp cao. Những ưu thế đó của lối nói hàm ẩn cũng thể hiện nét văn hóa giao tiếp của con người.

Hư từ trong ngôn ngữ nói chung và trong tiếng Việt nói riêng, tuy không thực hiện được

*Giáo sư, Tiến sĩ - Trường Đại học sư phạm Hà Nội 
chức năng định danh, không đảm nhiệm các chức năng ngữ pháp chính trong cụm từ và trong câu, nhưng đối với việc thể hiện nghĩa tình thái trong câu và đối với việc tạo lập câu mang nghĩa hàm ẩn thì nó lại là một phương tiện có giá trị không nhỏ. Báo cáo phân tích vai trò của một số lớp hư từ thuộc phụ từ, tình thái từ và quan hệ từ trong tiếng Việt đối với việc thể hiện nghĩa hàm ẩn trong câu, hơn nữa phần nghĩa hàm ẩn đó có thể thuộc lĩnh vực nghĩa miêu tả hay nghĩa tình thái. Qua đó góp phần khẳng định vai trò của hư từ tiếng Việt trong văn hóa giao tiếp bằng ngôn ngữ của người Việt.

1. Như đã biết, trong giao tiếp ngôn ngũ̃, nghĩa của câu (phát ngôn) thường được phân biệt theo hai tiêu chí: a) theo tính chất của nghĩa: nghĩa miêu tả/nghĩa tình thái; b) theo phương thức biểu hiện: nghĩa tường minh/nghĩa hàm ẩn.

Nghĩa miêu tả là nghĩa đề cập đến sự việc (sự tình) trong hiện thực (hoạt động trạng thái, quá trình, quan hệ...), còn nghĩa tình thái thì có một phạm vi rộng, trong đó rất quan trọng là phần liên quan đến điểm nhìn, sự đánh giá của người nói đối với sự việc đó. Nghĩa miêu tả mới chỉ là ngôn liệu, từ đó có thể được tình thái hóa với nhiều sắc thái khác nhau tạo nên những phát ngôn vừa có phần xương thịt, vừa có phần linh hồn. ${ }^{1}$

Ở phương diện khác, nghĩa tường minh là nghĩa hình thành từ nghĩa của các từ ngũ và kết cấu ngữ pháp của câu, không phụ thuộc vào ngữ cảnh và không cần đến hoạt động suy ý của người nghe, người đọc. Trái lại nghĩa hàm ẩn là phần nghĩa cần có sự suy ý của người nghe, người đọc trên cơ sở nghĩa tường minh và ngữ cảnh sử dụng. Nó là phần "ý tại ngôn ngoại”, nhưng nhiều khi lại là phần quan trọng nhất mà người nói muốn gửi đến người nghe. Việc sử dụng ngôn ngữ mang nghĩa hàm ẩn chẳng những làm cho lời nói ngắn gọn, súc tích, lại có tính lịch sự, tôn trọng trí tuệ, sự suy ý của đối tác giao tiếp, hơn nữa còn có thể tăng cường hiệu lực giao tiếp so với lối nói tường minh, hiển ngôn. Đó chính là một biểu hiện của văn hóa giao tiếp bằng ngôn ngữ của con người. Trong tiếng Việt có nhiều phương tiện và cách thức tạo nên những lời nói có nghĩa hàm ẩn, trong số đó có việc dùng hư từ. Báo cáo này tập trung vào việc xem xét giá trị của hư từ trong việc mã hóa nghĩa hàm ẩn và cũng là cơ sở giải mã nghĩa hàm ẩn. Từ đó thấy rõ hơn nét văn hóa trong giao tiếp ngôn ngữ của người Việt .

2. Hư từ trong tiếng Việt có thể được phân biệt thành phụ từ, quan hệ từ và tình thái từ. Đối với việc cấu tạo những phát ngôn mang nghĩa hàm ẩn thì phụ từ và tình thái từ có tần số sử dụng cao và có những sắc thái tinh vi tế nhị .

2.1. Các phụ từ không chỉ bổ sung ý nghĩa cho các thực từ để hoàn chỉnh nghĩa tường minh của câu, mà còn là phương tiện hữu hiệu trong việc thể hiện nghĩa hàm ẩn. Chẳng hạn, nhóm phụ từ chỉ ý nghĩa đồng nhất luôn luôn tiền giả định hai đối tượng đồng nhất. Khi cả hai đối tượng đều được hiển lộ trong câu thì có thể câu chỉ có nghĩa tường minh. Nhưng khi một hay cả hai đều vắng mặt trong câu thì câu có phần nghĩa hàm ẩn và phụ từ đồng nhất chính là tâm điểm ngụ chứa nghĩa hàm ẩn. Hãy xem xét một số ví dụ :

(1) Môn toán và môn văn, nó đều học giỏi.

(lời nói hàng ngày)

(2) Mẹ vẫn đào hầm. (lời bài hát)

(3) Ngày trước làm quan cũng thế a ?

(Nguyễn Khuyến)

${ }^{1}$ Bùi Minh Toán

a) Câu trong hoạt động giao tiếp tiếng Việt, GD, 2012, tr.63-127

b) Hư từ tiếng Việt - tiếp cận tích hợp từ lý thuyết ba bình diện, TCTĐH\&BKT, số 3/2013. 
Trong câu (1) từ đều cho biết có mối quan hệ đồng nhất được biểu hiện và đó là sự đồng nhất của hai đối tượng mà cả hai hiện diện trong câu (môn toán, môn văn). Nhờ sự có mặt của từ đều và những từ thể hiện hai đối tượng đó mà ý nghĩa đồng nhất được thể hiện tường minh trong câu này: hai đối tượng (môn toán và môn văn) đồng nhất ở phương diện được nó "học giỏi”.

Ở câu (2), dùng từ vẫn thể hiện ý nghĩa đồng nhất nhưng các đối tượng đồng nhất không hiện diện trong câu. Khi lĩnh hội, tất yếu ở người nghe, người đọc nảy sinh câu hỏi: từ vẫn mang vào câu lượng thông tin gì ? hay cụ thể hơn: nó báo hiệu sự đồng nhất cái gì với cái gì ? Trong bản thân ý nghĩa của nó, từ vẫn tiền giả định sự đồng nhất về tham thể hoàn cảnh, nhất là hoàn cảnh thời gian: giữa quá khứ và hiện tại, giữa trạng thái đã qua và trạng thái hiện đương, giữa các hoàn cảnh khác nhau,...Tuy trong nội bộ câu "Me vẫn đào hầm" không có cả hai tham thể hoàn cảnh, nhưng văn cảnh sau đó trong lời ca tường minh hóa hai đối tượng đồng nhất: từ lúc "tóc còn xanh" đến "nay me đã pho pho đầu bạc". Còn khi chưa được tường minh hóa, nghĩa của câu có dùng từ vẫn có thể khúc giải thành hai phần: phần nghĩa tường minh + phần nghĩa hàm ẩn như sau:

\section{Mẹ vẫn đào hầm = Mẹ đào hầm + Việc đó diễn ra từ trước đến nay}

Ở câu (3), từ cũng báo hiệu có nghĩa hàm ẩn. Như các từ đều và vẫn, từ cũng luôn đòi hỏi sự đồng nhất của hai đối tượng. Nhưng trong câu chỉ có một đối tượng: đời trước. Còn một đối tượng khác thì người đọc cần suy ý: nói đời trước là để liên hệ đến thời gian đối lập của nó: đời nay (tức đương thời Nguyễn Khuyến). Câu thơ của Nguyễn Khuyến dựa trên văn cảnh (nói về chi tiết gia đình Thúy Kiều trong Truyện Kiều nhờ có ba trăm lạng lo lót cho quan mà được quan xử tha bổng trong vụ họ bị một kẻ bán tơ vu oan: Có tiền việc ấy mà xong nhỉ, Đời trước làm quan cũng thế $a$ ?) có thể khúc giải như sau :

Đời trước làm quan cũng thế $\mathrm{a}$ ? = Đời trước có việc quan ăn tiền khi xử kiện + việc như thế giống với đời nay sao ?

(đương thời Nguyễn Khuyến).

Từ đó suy ra nghĩa hàm ẩn sâu cay, chua chát trong lời thơ của Nguyễn Khuyến: Đời nay (đương thời Nguyễn Khuyến) cũng $n h u$ đời trước, việc đến cửa quan giải quyết cần phải có tiền mói xong được!

Vai trò của hư từ ở câu thơ trên trong việc ngụ chứa nghĩa hàm ẩn càng thấy rõ hơn nếu thay thế từ cũng bằng một hư từ khác trong khi giữ nguyên các từ ngữ còn lại. Khi đó, nghĩa và hàm ý của câu thay đổi nhiều. Chẳng hạn thay bằng từ lại: Có tiền việc ấy mà xong nhỉ, Đời trước làm quan lại thế $a$ ? Câu mới vẫn có nghĩa hàm ẩn nhưng là một nghĩa hàm ẩn khác xa nghĩa cũ: không đánh đồng đời trước với đương thời, mà đối lập (nhờ từ lại - cũng là một hư từ): ngạc nhiên, chỉ trích, lên án đời trước với thói tham nhũng ăn tiền xấu xa, tiêu cực của quan lại mà đương thời không có (?!). Rõ ràng chỉ một hư từ mà chất chứa bao nhiêu hàm ý của người nói.

Khi khảo sát các hư từ đã, rồi, đang, tác giả Cao Xuân Hạo lưu ý nghĩa hàm ẩn của chúng khác nhau khi chúng được dùng với các động từ (đúng ra phải là vị tù nói chung BMT) "tĩnh" hay "động”. Khi dùng với các động từ tĩnh (chỉ trạng thái hay tính chất), các từ đã, rồ "cho biết rằng các trạng thái được động từ đi sau biểu thị là hiện thực trong thời gian hiện tại (lúc đang nói hay trong một thời gian được chọn làm mốc trong quá khứ hay tương lai )”. Tác giả nêu ví dụ so sánh: Bác Tu khỏe/ Bác Tu khỏe rồi/ Bác Tu nay đã khỏe và đi đến nhận định "trong các câu có đã, rồi có 
thể thấy lộ ra một tiền giả định mà các câu không dùng đã, rồi không hề có: cái trạng thái được vị ngữ biểu thị bây giờ mới có, chứ trước kia thì nó chưa có”. Trái lại, khi dùng với các động từ "động" (chỉ sự việc - hành động hay quá trình) ví dụ: Tôi lấy tiền rồi Tôi đã lấy tiền rồi thì cho biết "việc lấy tiền đã diễn ra trước đó và khi nói hai câu này nó không còn diễn ra nữa".

Điều nhận định trên rất đáng lưu ý vì hướng mọi người đến một góc khuất trong nhận thức hiện thực xung quanh mà thể hiện bằng từ ngữ của người Việt. Nhưng nếu vận dụng rộng hơn đối với các động từ khác thì thấy cần suy xét thêm. Chẳng hạn, động từ chảy mang tính "động” rất rõ nhưng trong một câu mà nó làm vị ngữ với thành tố phụ là từ đã hay rồi (Nước đã chảy/Nước chảy rồi) thì cái quá trình chảy đúng là diễn ra trước thời điểm nói, nhưng có thể vẫn còn tiếp tục diê̂n ra trong lúc nói câu đó, chứ không phải "không còn diễn ra nũa". Vả chăng, trong tiếng Việt có những động từ mà việc mang tính "tĩnh" hay tính "động" còn phụ thuộc vào văn cảnh sử dụng (so sánh: Công nhân in sách - tính động/Sách in rất đẹp - tính tĩnh). Phải chăng sự khác biệt về phần nghĩa hàm ẩn khi dùng các hư từ đã, rồi, đang với các động từ trong câu không phụ thuộc vào tính tĩnh hay động của động từ, mà phụ thuộc vào điểm tính hay quảng tính của sự tình mà động từ biểu hiện?

Sau đó tác giả Cao Xuân Hạo còn đi đến một nhận định xa hơn về hàm ý của những câu dùng đã, rồi, đang với các động từ “động" và "tĩnh":

"Một động từ động dùng với đã, rồi (hay chura) chỉ một sự việc xảy ra trước khi nói (hay trước một thời điểm được chọn làm mốc), nhưng lại mang hàm ý về cái trạng thái hiện tại (hay trong thời điểm được chọn làm mốc) vốn là kết quả của sự việc ấy”. Tác giả minh họa nhận định qua việc so sánh các ví dụ:

a) Bàn lau rồi đấy > bàn sạch đấy; Bàn chura lau > bàn bẩn đấy

b) Ăn sáng rồi > đang no, không ăn nữa đâu; Chưa ăn sáng > đang đói, sẵn sàng đi ăn ${ }^{2}$ (Kí hiệu > đọc là "có hàm ý”, ví dụ: "Bàn lau rồi đấy > Bàn sạch đấy" => "Bàn lau rồi đấy" có hàm ý "bàn sạch đấy”). Còn theo tác giả, dùng đang với các động từ tĩnh lại thêm cho động từ ấy một hàm ý là trạng thái tính chất ấy có thể sẽ có lúc kết thúc. Theo đó, câu "Nam đang ở Hà Nội” thể hiện một trạng thái nhất thời mà sau đó có thể thay đổi (so với câu Hồ Gươm $o^{3}$ Hà Nội). Nhận định như thế về hàm ý khác nhau liên quan đến việc dùng các từ đã, rồi, đang với động từ động hay tĩnh là những nhận định tinh tế, thú vị. Tuy nhiên cần bổ sung vào sự lý giải của tác giả điều kiện về ngữ cảnh bởi vì mỗi hàm ý đều quan hệ mật thiết với ngữ cảnh sử dụng. Chẳng hạn, cũng câu a trên (Bàn lau rồi đấy), nhưng trong ngữ cảnh tiếp viên nhà hàng nói rồi chuẩn bị ra về thì nó có thể lại mang hàm ý: "Tôi làm xong việc và bây giờ hoàn toàn có thể ra về".

Như thế chính các từ đã, rồi, chưa, đang khi dùng trong câu có thể ngụ chứa những nghĩa hàm ẩn (tiền giả định, hàm ý) mà người nói không muốn hay không cần thiết nói ra một cách tường minh, còn người nghe có thể suy ra và lĩnh hội tùy thuộc vào ngữ cảnh sử dụng.

Ở tất cả các trường hợp nói trên đây, hai phần nghĩa tường minh và hàm ẩn (gồm tiền giả định và hàm ý) đều thuộc về nghĩa miêu tả của câu. Nhưng các phụ từ còn có thể được dùng với một nghĩa tình thái hàm ẩn, trong đó có tình thái đánh giá của người nói. Chẳng hạn, từ mới, từ đã không chỉ là các phụ từ chỉ

${ }^{2}$ Cao Xuân Hạo (đồng tác giả), Tiếng Việt 12, Ban KHXH, GD, 1995, tr. 109-117. 
thời thể, để bổ sung nghĩa cho các thực từ (chẳng hạn: Nó mói về. Nó đã về), mà còn có thể hàm ẩn một nghĩa tình thái. Với một câu (4) như "Bây giờ đã là tháng 8 rồi" và ngược lại là câu (5) "Bây giờ mới tháng 8 thôi" ta có thể khúc giải thành hai phần nghĩa tường minh và hàm ẩn, trong đó phần tường minh là nghĩa miêu tả, phần hàm ẩn là nghĩa tình thái (tình thái đánh giá):

Bây giờ đã là tháng 8 rồi = Bây giờ là tháng $8+$ Tôi (người nói) cho nhu thế là muộn, không còn nhiều thòi gian nũa .

Bây giờ $m$ ới tháng 8 thôi = Bây giờ là tháng $8+$ Tôi (người nói) cho nhu thế là sớm, còn nhiều thòi gian nũa.

Các phụ từ chỉ lượng (nhũ̃ng, chỉ, có, độc,...) hay mức độ (đến, tới, tận...) cũng có thể đánh dấu nghĩa hàm ẩn về tình thái. Xét các câu:

(6) Thiếu nhũ̃ng 18 thằng kia à? (Nguyễn Công Hoan)

(7) Tôi chi ốm có một trận đấy thôi

(Nam Cao)

Nghĩa của các câu có thể khúc giải thành hai phần: phần nghĩa tường minh thuộc nghĩa miêu tả và phần nghĩa hàm ẩn thuộc nghĩa tình thái, như sau :

Thiếu những 18 thằng kia à? = Thiếu 18 thằng + Tôi (lý trưởng) cho số luọng nhu thế là quá nhiều

Tôi chỉ ốm có một trận đấy thôi! = Tôi ốm một trận + Tôi (lão Hạc) cho rằng trận ốm ấy là duy nhất, it ỏi (so với hậu quả mà nó gây ra).

Không chỉ hàm ẩn nghĩa đánh giá về mặt lượng như các trường hợp trên, câu có dùng phụ từ còn có thể hàm ẩn tình thái đánh giá về chất đối với tham thể hay sự tình được nói đến. Xét các câu tục ngữ có từ cũng sau :

(8) Kiến tha lâu cũng đầy tổ.

(9) Giặc đến nhà, đàn bà cũng đánh.
Từ cũng trong các câu này hàm ẩn một sự đồng nhất: con kiến tuy bé nhỏ nhưng nếu chăm chỉ (tha lâu) thì chẳng thua kém gì nhũng loài vật to lớn, mạh mẽ khác; đàn bà thường bị quan niệm là phái yếu, nhưng khi giặc đến nhà thì họ đánh giặc chẳng thua kém gì nhũng người đàn ông khỏe manh. Như thế, ngoài hàm ẩn nghĩa về sự đồng nhất, mỗi câu tục ngữ trên, với việc dùng từ cũng, còn hàm ẩn nghĩa tình thái đánh giá: đánh giá cao các đối tượng kiến, đàn bà với nhũng phẩm chất tốt đẹp.

Cũng có khi là sự đánh giá tiêu cực. Ví dụ:

(10) Nó là em tôi mà cái gì cũng giành

(Nguyễn Thi)

(11) $\hat{E}$, bán con chim gì đó mày?

- Con điêng điểng mà cũng không biết.

(Đoàn Giỏi)

Câu (10) hàm ẩn nghĩa đánh giá tiêu cực đối với người em, chê trách em: em mà không khác gì kẻ xa lạ - tranh giành với chị mọi thứ. Câu (11) với việc dùng từ cũng hàm ý chê kẻ hỏi: con điêng điểng là con vật thông thường như nhiều con vật khác mà kẻ đó không biết thì hiểu biết thật đáng bị coi thường!

Qua các ví dụ và sự phân tích ở trên có thể nhận định: phụ từ tiếng Việt có thể đảm nhiệm chức năng làm dấu hiệu cho nghĩa hàm ẩn, hơn nữa nghĩa hàm ẩn đó có thể thuộc phần nghĩa miêu tả mà cũng có thể thuộc phần nghĩa tình thái trong câu.

2.2. Bây giờ ta xem xét đến tình thái tù̀ trong chức năng thể hiện nghĩa hàm ẩn. Xét ví dụ sau:

(12) "Một hôm tôi phàn nàn việc ấy (việc "Lão từ chối tất cả những cái gì tôi cho lão" BMT) với Binh Tư... Hắn bĩu môi và bảo:

- Lão làm bộ đấy! Thật ra thì lão chỉ tâm ngẩm thế, nhưng cũng ra phết chứ chả vừa đâu..." (Nam Cao) 
Trong ví dụ trên có hai từ vốn xuất thân từ các đại từ, đã hư hóa thành hư từ thuộc loại tình thái từ cuối câu: đấy và đâu. Khi là thực từ, đấy chỉ định vị trí không gian, hoặc thời gian, hay sự vật ở xa người nói hoăc xa thời điểm nói, còn đâu dùng để hỏi hay phiếm định về sự vật hay vị trí không gian.

Trong tư cách tình thái từ ở cuối câu trên đây, từ đấy vừa giữ nét nghĩa: sự việc không phải ở trước mắt hay ngay lúc đang nói (việc lão Hạc từ chối sự giúp đỡ của ông giáo đã xảy ra từ trước), vừa có sắc thái cảnh báo để người nghe chú ý mà cảnh giác, để đừng nghĩ rằng lão hiền lành chân thật. Với ý nghĩa đó, có thể khúc giải câu trên như sau :

Lão làm bộ đấy = Lão làm bộ + anh (người đối thoại) đùng tưởng lão làm nhũng việc tù̀ chối bấy lâu nay là chân thật .

Còn từ đâu vừa nhấn mạnh ý phủ định, vừa bác bỏ quan niệm trái ngược về sự việc nói đến trong phát ngôn đi trước (quan niệm lão Hạc là người "vừa" - hiền lành, chất phác...). Có thể khúc giải như sau :

Lão... cũng ra phết chứ chả vừa đâu $=$ Lão... cũng ra phết chứ chả vừa $+a n h$ (người đối thoại) đùng nghĩ rằng lão hiền lành, chất phác

Gần với từ đấy là một số từ khác xuất thân là đại từ nhưng chuyển hóa thành hư từ tình thái: đây, kia, này... Khi chuyển thành hư từ chúng vẫn bảo lưu một số nét nghĩa vốn có. Chẳng hạn từ kia, khi là đại từ chỉ định, nó chỉ vị trí ở xa người nói (chỗ kia, cây kia...) hay thời gian xa so với thới điểm nói (hôm kia, năm kia...), còn khi là tình thái từ nó thể hiện nét nghĩa mức độ cao, xa hay nhiều. Chính vì thế, ở ví dụ (6) dẫn trên đây (Thiếu những 18 thằng kia à ?) từ kia phối hợp với từ nhũng đều thể hiện tình thái đánh giá của người nói đối với số lượng 18: cho rằng số lượng đó là cao, là quá nhiều.
Từ một số ngữ liệu như trên có thể thấy: cũng như phụ từ, tình thái từ trong tiếng Việt là những phương tiện ngụ chứa những nghĩa hàm ẩn (tiền giả định hoặc hàm ý) thuộc lĩnh vực nghĩa miêu tả và cả lĩnh vực nghĩa tình thái trong phát ngôn

2.3. Đối với các hư từ thuộc từ loại quan hệ từ thì có những nhóm quan hệ từ mà các thành viên có chức năng nghĩa cơ bản (thuộc lĩnh vực nghĩa miêu tả) giống nhau chỉ khác nhau về sắc thái biểu cảm, về sự đánh giá của người nói đối với sự tình được đề cập đến. Khi dùng trong câu, mỗi thành viên của nhóm sẽ làm cho câu có một nghĩa tình thái khác nhau ẩn chứa ở mỗi quan hệ từ được dùng. So sánh:

(13)... nếu đằng nhà gái họ cứ khăng khăng đòi như vậy, thì dẫu có bán vườn đi cũng không đủ cưới. (Nam Cao)

(14)... lão già yếu lắm rồi, không biết sống chết lúc nào, con không có nhà, lỡ chết không biết ai đứng ra lo cho được... (Nam Cao)

(15) Ngộ nó không lấy gì lo được, lại bán vườn thì sao? (Nam Cao)

(16) Giá cứ thế này mãi thì thích nhỉ?

(Nam Cao)

Như có thể thấy qua các ví dụ trên đây, nhóm hư từ nếu, lơ, ngộ, giá... đều có chức năng nghĩa cơ bản giống nhau: biểu hiện giả thiết (sự tình đi sau chúng chưa xảy ra trong thực tế, mới chỉ là giả thiết), nhưng hàm chứa nét nghĩa tình thái đánh giá khác nhau đối với sự tình được giả thiết: Từ nếu cho biết tình thái trung hòa, các từ $l \tilde{o}, n g \hat{o}$ - tình thái đánh giá tiêu cực, còn giá - tình thái đánh giá tích cực. Các nghĩa tình thái khác nhau này đều hội tụ ngầm ẩn trong các quan hệ từ, và khi quan hệ từ được dùng trong câu, chúng hàm chứa trong nghĩa của câu thành phần tình thái đánh giá của chủ thể lời nói. Cũng có thể khúc giải 
nghĩa của mỗi câu, như câu 15 , thành hai thành phần nghĩa dựa trên từ mang tình thái trung hòa (nếu) như sau:

Ngộ nó không lấy gì lo được lại bán vườn thì sao = Nếu nó không lấy gì lo được + (tôi cho rằng khả năng đó là điều xấu, tệ hại) thì sao?

So sánh thêm về nghĩa tình thái khác nhau ngụ chứa trong các quan hệ từ đồng nhất về nghĩa miêu tả: đánh dấu vai nghĩa nguyên nhân (vì, do, bởi, tại, nhờ), nhưng khác nhau về tình thái đánh giá đối với nguyên nhân:

(17) Bà Hân mắt đã mờ đi vì khóc

(Hồ Anh Thái)

(18) Tôi hồi phục sức khỏe rất nhanh, chủ yếu $d o$ trẻ trung, chẳng phải $d o$ thuốc

(Nguyễn Huy Thiệp)

(19) Nguyễn Thị Lộ lo lắng bởi những đổi thay trong tinh thần Nguyễn

(Nguyễn Huy Thiệp)

(20) Chắc tại chị không chịu kiếm tiền mà cứ lo học nên mới nghèo

(Hồ Anh Thái)
(21) Mẹ thằng Bính cũng nhờ ăn gạo ấy mà đẻ nó ra thực chóng dễ như trở bàn tay, anh ạ. (TNVN tuyển chọn)

3. Tóm lại, đối với nghĩa hàm ẩn của phát ngôn, hư từ tiếng Việt (cả phụ từ, tình thái từ và quan hệ từ) có tiềm năng làm dấu hiệu cho các nghĩa hàm ẩn thuộc lĩnh vực nghĩa miêu tả hoặc nghĩa tình thái. Nhờ các hư từ bé nhỏ mà câu tuy có hình thức ngắn gọn nhưng hàm chứa nhiều nội dung ý nghĩa sâu xa, tế nhị. Người nói không cần thiết phải "nói trắng ra", mà dựa vào ngữ cảnh và dành một phần cho sự khám phá và lĩnh hội của người đối thoại. Với việc dùng hư từ một cách thích hợp, nhưng linh hoạt, sáng tạo, sự giao tiếp ngôn ngữ giữa người và người chẳng những đạt được mục đích nhận thức, thông tin mà còn gia tăng rất nhiều chất văn hóa, lịch sự, và cả hiệu quả giao tiếp ngôn ngữ. 